\title{
Konzepte gegen die Krise? Chancen und Ambivalenzen betrieblicher "Besser"-Strategien für Arbeitspolitik und Interessenvertretungen
}

\author{
Antonio Brettschneider/Tabea Bromberg \\ Thomas Haipeter/Steffen Lehndorff
}

\begin{abstract}
Aktive Arbeitspolitik gilt als vielversprechendes Zukunftsfeld für die Interessenvertretungspolitik. Die Entwicklung und Aushandlung innovativer Arbeitskonzepte für eine umfassende und nachhaltige Nutzung der Qualifikations- und Kooperationspotenziale der Beschäftigten scheint den Interessenvertretungen Möglichkeiten zu eröffnen, aus ihrer Defensive heraus wieder stärker in die Offensive zu kommen. Der Artikel untersucht, welche Erfahrungen dazu mit der arbeitspolitischen Initiative „Besser statt billiger“ in der nordrheinwestfälischen Metallindustrie gemacht werden. Es zeigt sich, dass „Besser“-Konzepte tatsächlich gute Chancen für eine offensive Politikgestaltung bieten können. Sie bleiben aber an Defensivkonstellationen gebunden, erfordern eine neue Interessenvertretungspraxis und sollten der Frage guter Arbeitsbedingungen einen höheren Stellenwert einräumen.
\end{abstract}

\section{Interessenvertretungen und die Re-Aktivierung der Arbeitspolitik}

Vor mittlerweile zehn Jahren hat Michael Schumann die These formuliert, dass die betrieblichen Rationalisierungswellen der 1980er und 1990er Jahre neue Unübersichtlichkeiten in der Arbeitspolitik geschaffen hätten. Zum einen komme es zu einer Ausdifferenzierung der Problemlagen einzelner Beschäftigtengruppen, insbesondere zwischen prekärer Beschäftigung und konventioneller Arbeitspolitik auf der einen und innovativer Arbeitspolitik und Wissensarbeit auf der anderen Seite. Und zum anderen träten neue Widerspruchsmomente innerhalb innovativer Arbeitsformen zutage. Auch innovative Arbeitspolitiken, verstanden als umfassende Nutzung und Entwicklung der Qualifikationen der Beschäftigten, ihrer Kooperationsfähigkeiten und ihrer Mitsprachemöglichkeiten, paarten sich zunehmend mit steigenden Leistungsanforderungen und wachsendem Marktdruck (Schumann 2001).

Die fortschreitende Dominanz des Finanzmarktkapitalismus schafft nach Schumann jedoch mittlerweile wieder neue Übersichtlichkeiten (Schumann 2008), weil sie Arbeitnehmer und Interessenvertretungen in eine machtpolitische Defensive zwingt. Beschäftigungspolitisch sei deshalb mit weiterer Prekarisierung zu rechnen, und leistungspolitisch stehe eine weitere Aushöhlung der Arbeitszeit- und Leistungsnormen an. Gewerkschaften müssten deshalb eine Abwehr- und Schutzpolitik betreiben. In der Arbeitspolitik hingegen seien weiterhin vielfältige betriebliche Konzepte erkennbar. Interessenvertretungen sollten daher aktiv für eine innovative Arbeitspolitik in den Unternehmen kämpfen. Erfolge auf diesem Feld könnten ihnen zugleich die Zustimmung der Beschäftigten sichern.

Diese Aufforderung zu einer Re-Aktivierung der Arbeitspolitik stößt in den Gewerkschaften durchaus auf offene Ohren. In der IG Metall beispielsweise werden sowohl auf Vorstandsebene als auch in mehreren Bezirken seit einigen Jahren verschiedene arbeitspolitische Projekte entwickelt und umgesetzt. Eine der ersten und wichtigsten Initiativen in diese Richtung wurde mit der Kampagne „Besser statt billiger" entwickelt, die der Bezirk NRW der IG Metall im Jahr 2005 startete (Korflür et al. 2010). Im Zentrum der Kampagne steht die gewerkschaftliche Aktivierung der Betriebsräte für die Erarbeitung und Aushandlung betrieblicher „Besser“Strategien. Betriebsräte sollen in die Lage versetzt werden, Unternehmensforderungen nach Tarifabweichungen, Ver- oder Auslagerungen, Personalabbau oder einer Re-Taylorisierung der Arbeitsorganisation eigene Konzepte einer zukunftsfähigen und beschäftigungssichernden Unternehmenspolitik entgegenzusetzen. Die gewerkschaftlichen Unterstützungsleistungen sind dabei breit gefächert. Sie reichen von der gemeinsamen Verhandlung von Tarifabweichungen über die Organisation externer gewerkschaftsnaher Beratung bis hin zur Ausrichtung themenzentrierter Workshops oder der Koordinierung wechselseitiger Unterstützung zwischen den Betriebsräten in Form von Branchennetzwerken. Die Auseinandersetzung um bessere Konzepte wird bei Verhandlungen von Tarifabweichungen besonders virulent (zu den Herausforderungen von Tarifabweichungen für Betriebsräte siehe Haipeter 2010). In diesen Fällen sollten keine Konzessionen mehr ohne Gegenleistungen wie Beschäftigungssicherung, Investitionszusagen, Innovationsprogramme oder Qualifizierungsvereinbarungen gewährt werden. Aber auch jenseits der Defensivkonstellationen von Tarifabweichungen sollen „Besser“-Konzepte proaktiv entwickelt und eingefordert werden mit dem

Antonio Brettschneider, Institut Arbeit und
Qualifikation (IAQ), Universität Duisburg-
Essen. Arbeitsschwerpunkte: Sozialpolitik,
Vergleichende Wohlfahrtsstaatenforschung,
Industrielle Beziehungen.
e-mail: antonio.brettschneider@uni-due.de
Tabea Bromberg, IAQ, Universität Duisburg-
Essen. Arbeitsschwerpunkte: Industrielle Be-
ziehungen, Arbeits- und Produktionssysteme.
e-mail: tabea.bromberg@uni-due.de
Thomas Haipeter, PD Dr., IAQ, Universität
Duisburg-Essen. Arbeitsschwerpunkte: Indus-
trielle Beziehungen, Industriesoziologie.
e-mail: thomas.haipeter@uni-due.de
Steffen Lehndorff, Dr., IAQ, Universität
Duisburg-Essen. Arbeitsschwerpunkte:
Arbeitszeit, Industrielle Beziehungen,
Beschäftigungsmodelle im Vergleich.
e-mail: steffen.Iehndorff@uni-due.de


Ziel, für nachhaltige Organisations- und Innovationsstrategien zu kämpfen und die Interessenvertretungen wieder in eine Position der Offensive zu bringen.

\section{Neue Unübersichtlich- keiten in der Krise}

Die Entwicklungen und Auswirkungen einer aktiven Arbeitspolitik der Interessenvertretungen können anhand betrieblicher „Besser“-Konzepte empirisch überprüft werden, und dies wollen wir im vorliegenden Beitrag anhand eigener, allerdings noch vorläufiger Forschungsergebnisse versuchen. ${ }^{1}$ Dabei ist davon auszugehen, dass sich mit der aktuellen Finanzmarktkrise die Unübersichtlichkeiten in den arbeits- und interessenvertretungspolitischen Problemlagen wieder vergrößert haben könnten. Auf der einen Seite ist der überraschende Befund festzuhalten, dass Gewerkschaften und Betriebsräte in der Krise eine neue Anerkennung zu erfahren scheinen. Auf Makroebene wurden die Gewerkschaften im Rahmen eines Krisenkorporatismus in die Entwicklung staatlicher Maßnahmen zur Krisenbewältigung einbezogen. Auf Mikroebene sind die Betriebsräte in betrieblichen Notgemeinschaften wichtige Partner beim Umgang mit den Krisenfolgen und bei der Organisation der Arbeitszeitverkürzung durch Kurzarbeit, Beschäftigungssicherungsvereinbarungen oder dem Abbau von Arbeitszeitkonten (dazu Seifert 2010). Um qualifizierte Fachkräfte zu halten, haben die Unternehmen sogar - abweichend von früheren Krisenverläufen - erhebliche Einbrüche der Arbeitsproduktivität in Kauf genommen (Herzog-Stein 2010). Sofern sich dahinter eine neue Bedeutung der Nutzung von Qualifikationen für die Unternehmen verbirgt, könnte sich mit der Krise tatsächlich ein Fenster für arbeitspolitische Innovationen geöffnet haben.

Die hier aufblitzenden Chancen für eine qualifikationsbasierte Arbeitspolitik sollten sehr ernst genommen werden, aber blauäugiger Optimismus ist unangebracht. Denn auf der anderen Seite werden die Weichen in Richtung auf Restrukturierung und strategischen Beschäftigungsabbau in vielen Bereichen der Industrie bereits gestellt. Auch die Aufwertung der Interessenvertretungen könnte sich als eine lediglich temporär wirksame „geliehene Macht" erweisen (Ehlscheid et al. 2010), die endet, sobald ihre Fähigkeiten als Ordnungsmacht oder Krisenmanager nicht mehr gebraucht werden. Mehr noch, auch aufseiten der Unternehmen ist der finanzmarktgetriebene Steuerungsmodus offensichtlich ungebrochen. Eine Legitimationskrise des Finanzmarktkapitalismus in den Unternehmen ist nicht erkennbar; mit den Banken ist auch ihre Ideologie gerettet worden (Boyer 2010). Insbesondere können die „Bargaining Chips“ (Dörre/Holst 2009) dieser Steuerungsformen in Gestalt von Aus- und Verlagerungsdruck als Drohszenarien weiterhin genutzt werden, um Arbeitsstandards infrage zu stellen und Interessenvertretungen gefügig zu machen. Grundsätzlich ist ferner zu bedenken, dass die altbekannten Krisensymptome der Interessenvertretungen wie die wachsende Fragmentierung abhängig Beschäftigter und das Schwinden betrieblicher Organisationsmacht in der Krise nicht an Bedeutung eingebüßt haben (Crouch 2010).

Ein Automatismus der Stärkung innovativer Arbeitspolitiken und mit ihnen der Interessenvertretungen ist mithin nicht $\mathrm{zu}$ erwarten. Eine aktive Arbeitspolitik scheint mehr als zuvor unverzichtbare Voraussetzung für arbeitspolitische Fortschritte (so auch Kuhlmann 2009). Allerdings wären damit die Ambivalenzen einer innovativen Arbeitspolitik zwischen Autonomie und Qualifikationsnutzung auf der einen und Leistungsintensivierung und Marktdruck auf der anderen Seite noch nicht gelöst. Gerade in und nach der Krise ist nicht davon auszugehen, dass sich innovative Arbeitspolitik von den Abwehrkämpfen lösen lässt, in denen die Interessenvertretungen hinsichtlich des Erhalts der Arbeitsstandards gefangen sind. Zugleich könnte die Verteidigung der Arbeitsstandards aber auch zu einem wichtigen Auslöser für Auseinandersetzungen um innovative Arbeitspolitik werden.

Diese Punkte wollen wir im Folgenden am Beispiel der Kampagne „Besser statt billiger" und der Entwicklung und Aushandlung betrieblicher „Besser“-Strategien diskutieren. Wir lassen uns dabei vom Forschungsinteresse an neuen Ansätzen gewerkschaftlicher Praxis leiten und möchten zur Umorientierung vom „Krisen- zum Strategieparadigma“ (Urban 2008) in der Gewerkschaftsforschung beitragen. Welche Potenziale, so fragen wir, hat dieser arbeitspolitische Ansatz für die
Entwicklung einer innovativen Arbeitspolitik? Welche Konsequenzen sind damit für die Arbeit der Interessenvertretungen als zentrale Akteure der Arbeitspolitik verbunden? Und auf welche Weise können die Interessenvertretungen die Arbeitspolitik für die Stärkung der eigenen Verhandlungs- und Organisationsmacht nutzen?

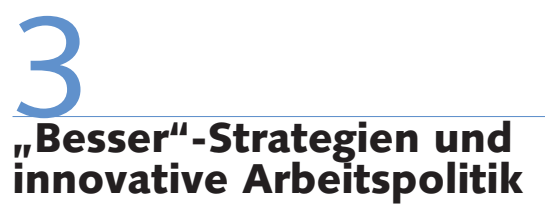

\subsection{BANDBREITE INNOVATIVER ARBEITSPOLITIK}

Was heißt eigentlich „besser“? Und haben solche „Besser“-Konzepte überhaupt etwas mit innovativer Arbeitspolitik zu tun? Zunächst einmal ist festzuhalten, dass die Bandbreite dessen, was in den untersuchten Fällen jeweils als „Besser“-Konzept entwickelt und verhandelt wurde, überaus groß ist. Dies lässt sich an drei Fällen darstellen. Ein im Sinne aktiver Arbeitspolitik defensives Verständnis von „Besser“ findet sich im Fall eines Automobilzulieferers, der im Besitz eines Finanzinvestors war und in großen Liquiditätsschwierigkeiten steckte. Der Erhalt der Produktionsarbeitsplätze stand im Zentrum der Auseinandersetzungen zwischen Betriebsrat und Management. Der Verlagerungsdruck in ein bestehendes osteuropäisches Produktionswerk war und ist groß; der deutsche Produktionsstandort wurde vor allem noch für kompliziertere Produktabläufe genutzt. Hier galt dem Betriebsrat schon die Sicherung des deutschen Produktionsstandortes als „Besser“-Konzept. An der Verbesserung der Produktionsabläufe

\footnotetext{
Wir stützen uns auf Zwischenergebnisse des von der Hans-Böckler-Stiftung geförderten Forschungsprojekts "Rückenwind für die Betriebsräte“, das Expertengespräche, Fallstudien und eine Befragung der Verwaltungsstellen des Bezirks NRW der IG Metall umfasst. Für unsere Analyse können wir das Material mehrerer Expertengespräche sowie zwölf Fallstudien nutzen, die abgeschlossen oder aktuell in Bearbeitung sind. Es sind Unternehmen unterschiedlicher Einzelbranchen und Betriebsgrößen, die jeweils Schauplatz von Konflikten um Besser-Konzepte waren oder sind Wir führen im Projekt insgesamt zehn Kurzfallstudien mit Interviews von Betriebsräten und gewerkschaftlichen Betreuern sowie sechs Intensivfallstudien mit zusätzlichen Interviews von Management und Vertrauensleuten oder Beschäftigten durch.
} 
im Sinne einer innovativen Arbeitspolitik arbeitete der Betriebsrat nicht. Vielmehr führte er an, dass er durch flexible Samstagsschichten sowie die Absenkung des Akkordsatzes und der übertariflichen Bezahlungen aktiv dazu beigetragen habe, eine Verlagerung zumindest größerer Produktionsumfänge zu verhindern. In seinen Deutungen sind „Besser“ und „Billiger“ nicht klar getrennt.

Der zweite Fall steht für „Besser“Konzepte, die zwar offensiver sind, aber verglichen mit dem umfassenden Anspruch innovativer Arbeitspolitik doch eher kleinteilig und punktuell erscheinen. Auch in diesem Unternehmen der Stahlindustrie waren ebenfalls die wirtschaftlichen Probleme erheblich. Hier konnten die Interessenvertretungen im Rahmen der Verhandlungen über eine Tarifabweichung durchsetzen, dass eine gewerkschaftsnahe Unternehmensberatung den Betrieb genauer unter die Lupe nahm. Mit ihr zusammen entwickelte der Betriebsrat schließlich das Konzept regelmäßiger Kommunikationsrunden zwischen Betriebsrat und Geschäftsleitung zur Verbesserung des Kommunikationsflusses in der Organisation. Weil nach Analyse des Beratungsunternehmens insbesondere die vertikalen Kommunikationsflüsse gestört waren, wurden auf Initiative des Betriebsrates zu diesen Runden jeweils Beschäftigte als Experten für bestimmte Probleme hinzugeladen. Durch diesen direkten Kommunikationskanal zwischen Shop Floor und Leitung wurden die Mitsprachemöglichkeiten der Beschäftigten erheblich gestärkt. Weitergehende Veränderungen der Leitungsstrukturen oder der Arbeitsorganisation waren allerdings nicht Teil des Konzepts.

Der dritte Fall eines Maschinenbauunternehmens schließlich steht für eine offensive und umfassende „Besser"-Strategie. Hier sind „Besser“-Konzept und die Idealvorstellungen einer innovativen Arbeitspolitik erstaunlich deckungsgleich. Kern des Konzepts sind die Einführung sowie die kontinuierliche Koordinierung und Weiterentwicklung teilautonomer Gruppenarbeit in allen Bereichen des Unternehmens. Vor mehreren Jahren hatte sich die wirtschaftliche Situation des Unternehmens, abweichend vom Verlauf der Branchenkonjunktur, dramatisch verschlechtert. Auf einer Klausurtagung mit der Geschäftsleitung konnte der Betriebsrat die flächendeckende Einführung von Gruppenarbeit durchsetzen. Zugleich hatte er in früheren Pilotprojekten und unter Hinzuziehung eines gewerkschaftsnahen Partners die Erfahrung gemacht, dass Gruppenarbeit nur erfolgreich ist, wenn die Leistungskennziffern sorgfältig bestimmt werden und das Entgeltsystem angepasst wird. Er teilte das für die Produktion ausgegebene Wirtschaftlichkeitsziel, die Durchlaufzeiten zu reduzieren. Im Laufe der Jahre wurden die Kennziffern mehrfach verändert. Auch betreute der Betriebsrat die Gruppenarbeit im Steuerungskreis und durch Teilnahme an Gruppensitzungen aktiv. Und in der Finanzmarktkrise, die das Unternehmen mit einem Auftragseinbruch von fast $50 \%$ hart traf, wurde nicht nur das Gruppenkonzept vom Werkstatt- auf das Fließprinzip umgestellt; auch konnte der Betriebsrat ein breit angelegtes Qualifizierungsprogramm durchsetzen, mit dessen Hilfe sowohl die Einsatzflexibilität der Beschäftigten in der neuen Gruppenorganisation als auch in vielen Fällen ihre tarifliche Eingruppierung erhöht werden konnte.

\section{2 "BESSER “-STRATEGIEN ALS BARGAINING CHIP}

Die Beispiele zeigen, dass „Besser“-Konzepte häufig nicht die „reine Lehre“ einer innovativen Arbeitspolitik repräsentieren, sei es, weil sie dazu nicht umfassend genug sind, oder sei es, weil sie dazu zu defensiv sind. Die Defensive allerdings ist eine Erfahrung, die in allen Fällen hinter der Entwicklung dieser Konzepte steht. Es sind wirtschaftliche Krisen, aber vor allem Drohszenarien des Managements in Richtung Verlagerung von Produktion oder Unterschreitung tariflicher Arbeitsstandards, die für die Betriebsräte Impulse setzen, sich über Alternativkonzepte Gedanken zu machen und dazu die Hilfe der Gewerkschaft und gewerkschaftsnaher Berater hinzuzuziehen. „Besser“-Konzepte richten sich daher gegen „Billiger“-Strategien der Unternehmensleitungen. Dabei wird nicht immer der ganz große Wurf im Sinne einer innovativen Arbeitspolitik angedacht oder gewagt. Das muss aber noch lange nicht heißen, dass die arbeitspolitischen Initiativen der Betriebsräte deshalb wirkungslos wären. Oftmals bieten sie den Betriebsräten in den Verhandlungen trotzdem ihrerseits einen wichtigen „Bargaining Chip“, den sie dem Management entgegensetzen können und mit dem sie die Deutungshoheit des Managements zumindest infrage stellen können. Was am Ende in den Verhandlungen herauskommt, sind ohnehin nicht die Konzepte in Reinform, sondern Kompromisse, die die betrieblichen Machtverhältnisse widerspiegeln. Aber auch für die weniger umfassenden arbeitspolitischen Konzepte gilt, dass sie von den Betriebsräten in den Verhandlungen wirkungsvoll genutzt werden können, weil sie Defizite des Managements aufdecken und öffentlich machen und zugleich ein Stück „Augenhöhe“ schaffen. Dieser Punkt wurde von den Betriebsräten durchgängig betont.

\subsection{INNOVATION UND BETRIEBSRATSARBEIT}

In diesem Sinne werden die Betriebsräte in ihren Organisationen zu Promotoren der Innovation. Dabei lässt sich - ähnlich wie bei der Einschätzung der arbeitspolitischen Stoßkraft der Konzepte - feststellen, dass die Innovationsthemen sehr vielfältig sind. Naheliegenderweise beziehen sich die innovationspolitischen Stoßrichtungen der Konzepte vor allem auf Organisationsfragen. Dies ist in den angeführten Beispielen der Kommunikationsrunden oder der Gruppenarbeit deutlich geworden. Betriebsräte sind als Kenner ihrer Betriebe Spezialisten der Organisation und ihrer Abläufe, und dies spiegelt sich in den Innovationsthemen zu einem guten Teil wider. Dass in diesem Rahmen die Reorganisationstiefe der Konzepte ganz unterschiedlich sein kann, wurde bereits festgestellt.

Es gibt aber auch Fälle, in denen die Innovationsbemühungen der Betriebsräte sich auf Themen konzentrieren, die mit Betriebsräten nicht von vorneherein assoziiert werden. Ein Beispiel dafür findet sich bei einem Anlagenbauer, dessen untersuchter Betrieb inzwischen fast vollständig aus Angestellten - und unter ihnen vor allem aus Ingenieuren - besteht, nachdem die vormals dazugehörige Werkstatt gegen den Widerstand des Betriebsrates ausgelagert worden war. Seinerzeit hatte der Betriebsrat für eine enge Verzahnung von Entwicklung und Fertigung im Sinne einer wechselseitigen Befruchtung plädiert, konnte damit aber nur den Erhalt der Werkstatt aushandeln, nicht aber ihren Verkauf verhindern. Seitdem hat sich der Betriebsrat, der sich selbst zumeist aus Ingenieuren rekrutiert, zu einem Spezialisten für unternehmensstrategische Fragen entwickelt, der die häufig wechselnden Geschäftsleitungen mit umfassenden Alter- 
nativkonzepten konfrontiert. Im aktuellen Konflikt um Personalabbau in der Entwicklung hat der Betriebsrat eine Analyse zur strategischen Rolle der Entwicklung im Unternehmen erstellt, deren Inhalte von Beschäftigten und sogar Führungskräften in großer Einmütigkeit geteilt werden Ende des Konflikts noch offen.

Die arbeitspolitischen Initiativen haben die paradox anmutende Komponente, dass damit Betriebsräte zu Verteidigern des häufig zitierten - aber selten genauer untersuchten - deutschen Produktionsmodells werden (zur Diskussion siehe Lehndorff 2009). In der Literatur über die „Spielarten des Kapitalismus“ wird Deutschland gerne als Beispiel für eine Interessenkonstellation genannt, in der ,die Arbeitskräfte (bis hinunter zum Shop Floor) qualifiziert genug sind, um Innovationen vorzuschlagen, abgesichert genug sind, um Vorschläge zur Veränderung von Produkten oder Prozessen zu riskieren, die ihre Situation im Betrieb ändern könnten, und mit genug Autonomie in der Arbeit ausgestattet sind, um derartige Verbesserungen als eine Dimension ihrer Arbeitsaufgabe ansehen zu können" (Hall/Soskice 2001, S. 39). Die genannten Beispiele zeigen, dass damit die Wirklichkeit in vielen Unternehmen nicht getroffen wird. Zwar profitieren die Unternehmen in der Tat von den Qualitäten des deutschen Ausbildungssystems, doch weder schöpfen sie die dort vermittelten Qualifikationen wirklich aus, noch sorgen sie für ihre systematische Weiterentwicklung, noch nutzen sie das Expertenwissen der Beschäftigten für Innovationsprozesse. Es sind vielmehr die Interessenvertretungen, die in den Unternehmen mit Alternativkonzepten darauf drängen, die Möglichkeiten des deutschen Produktionsmodells ernst zu nehmen und die Wissens- und Flexibilitätspotenziale der Beschäftigten zu aktivieren. Und dies geschieht zumeist gegen den erklärten Widerstand der Unternehmensleitungen, die von kurzfristigen Gewinnorientierungen geleitet werden und zumeist die Senkung der Personalkosten im Auge haben.

Allerdings, und hierin liegt eine zweite Paradoxie, sind die „Besser“-Konzepte der Interessenvertretungen nicht frei von den Ambivalenzen, die auch für die innovative Arbeitspolitik benannt wurden. Zum einen stellt sich die Frage nach der Dauerhaftigkeit der eingeleiteten Maßnahmen. In vielen Betrieben läutet das (möglicherweise in der Zwischenzeit personell neu zusammengesetzte) Management alle zwei Jahre eine neue „Runde“ ein, indem es begründet oder nicht - mit Entlassungen oder Verlagerung droht. Die Unternehmen verzichten auf ihre „Bargaining Chips“ zu keinem Zeitpunkt. Die Betriebsräte sind deshalb als Akteure innovativer Arbeitspolitik dauerhaft gefordert. Es gibt keine arbeitspolitischen Ruhephasen, weil sich die Defensivkonstellationen nur wegen einiger arbeitspolitischer Erfolge nicht verflüchtigen.

Zum anderen - und damit zusammenhängend - aber besteht das Problem, dass die „Besser“-Konzepte in den Deutungen der Interessenvertretungen häufig nicht klar mit Fragen der Arbeitsbedingungen verknüpft werden. $\mathrm{Ob}$ arbeitspolitische Initiativen auch zu einer Verbesserung der Arbeitsbedingungen - und damit zu einer „guten Arbeit“ - führen, wird teilweise nicht gefragt. Häufiger werden arbeitspolitische Innovationen mit leistungspolitischen Verschlechterungen bezahlt. Dies ist in den Verhandlungen nicht der erklärte Wille der Betriebsräte, sondern des Managements, aber es zeigt, wie eng Offensive und Defensive in der Arbeitspolitik zusammenhängen. Und die Betriebsräte sind für solche Konzessionen anfällig, weil die Frage guter Arbeitsbedingungen für sie teilweise ein weißer Fleck ist. Nicht, dass es kein Problembewusstsein gäbe. So gab ein gewerkschaftlicher Betreuer zu bedenken: „Manchmal hat das natürlich den Anstrich, dass Besser-Strategien auch Rationalisierungselemente sind." Und nicht alle Kostensenkungsmaßnahmen müssen - z.B., wenn es um die Verbesserung der Energieeffizienz geht - bedenkliche Auswirkungen auf die Qualität der Arbeitsbedingungen haben. Vereinzelt konnten sogar Maßnahmen wie die Einführung von Gruppenarbeit angestoßen werden, die für die Qualität der Arbeit als förderlich eingeschätzt werden können. Einen systematischen Stellenwert hat diese Frage jedoch bislang nur selten in den „Besser“Konzepten. Dies kann auflängere Sicht zu einem Problem werden, denn auf Dauer werden die Beschäftigten wohl nur dann hinter „Besser“-Strategien stehen, wenn auch ihre Arbeitsbedingungen Berücksichtigung finden.

Die weißen Flecken sind aber ein Problem der Praxis, nicht des Konzepts. So betonen Schroth und Wetzel (2009, S. 8f.), dass „, Besser statt billiger ‘... kein wertfreier Ansatz der Innovationsförderung “ sei: „Wir geben der Qualität der Arbeitsbedingungen den Vorzug vor der Wirtschaftlichkeit. (...) Innovationen müssen daran gemessen werden, ob sie zum gesellschaftlichen Fortschritt beitragen." Dazu gehören, wie diese Autoren fortfahren, die Sicherung und Schaffung von Arbeitsplätzen, eine gute Qualität der Arbeit und gesellschaftlich nützliche sowie ökologisch verträgliche Produkte. Tatsächlich würde erst die Fundierung betrieblicher Verbesserungsprozesse in „guter Arbeit“ auf die Nutzung jener Potenziale abzielen, die dem deutschen Produktionsmodell zugesprochen werden (vgl. Kirner et al. 2010): z. B. die Nutzung von Erfahrungswissen für die Schaffung eines gesund erhaltenden Arbeitsumfelds, die Erhöhung des Dispositions- und Gestaltungsspielraums der Beschäftigten, die Erhöhung des Qualifikationsniveaus und seine breitere Nutzung, also letztlich die stärkere Beteiligung der Beschäftigten. Es bleibt zu analysieren, wie weit diese konzeptionellen arbeitspolitischen Überlegungen Eingang in die betriebliche Praxis finden können.

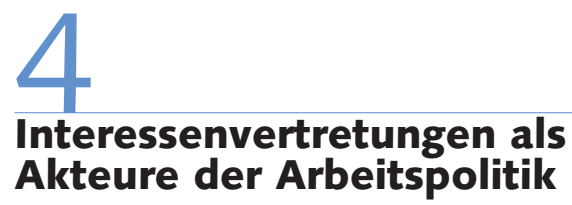

\subsection{NOTWENDIGE PRAXIS- VERÄNDERUNGEN}

Die Entwicklung und Umsetzung von Besser-Konzepten hat für die Betriebsräte grundlegende Konsequenzen. Es ist nicht damit getan, ein Alternativkonzept zu den Strategien des Managements zu entwickeln und dies zu verhandeln. Dies geht vielmehr mit weitgehenden Veränderungen und Umbrüchen in der Arbeit der Interessenvertretung einher. Hier lassen sich drei eng miteinander verbundene Entwicklungen feststellen: erstens eine stärkere (betriebs-)wirtschaftliche und strategische Orientierung der Betriebsräte, oftmals unter verstärktem Rückgriff auf externen Sachverstand und externe Beratung, zweitens Neuorganisationen im Betriebsratsgremium bei Professionalisierung seiner Mitglieder und drittens eine verstärkte Beteiligung und Einbeziehung der Beschäftigten.

Zunächst ist zu beobachten, dass Betriebsräte sich in den von uns untersuchten Fällen mit Grenzen ihrer bisherigen Praxis 
konfrontiert sehen. Dies gilt ganz unabhängig von ihren unterschiedlichen Traditionen betrieblicher Interessenvertretung und davon, ob sie eher sozialpartnerschaftlich oder eher konfliktorientiert agieren. Es sind Situationen in den Betrieben entstanden, in denen das Verständnis der Betriebsräte für betriebswirtschaftliche Zusammenhänge zur fundamentalen Voraussetzung für den Erhalt ihrer Handlungsfähigkeit wurde. Managemententscheidungen werden vermehrt mit betriebswirtschaftlichen Zwängen begründet und mit betriebswirtschaftlichen Kennzahlen unterfüttert. Teilweise geht - wie in einem der von uns untersuchten Betriebe - die Geschäftsführung deshalb von selbst dazu über, dem Betriebsrat von sich aus mehr betriebswirtschaftliche Daten zur Verfügung zu stellen, um sein Verständnis für die betriebswirtschaftliche Seite einer Entscheidung zu schärfen. Dieser Betriebsrat hat das Angebot dann übrigens offensiv für eigene Gestaltungsvorschläge genutzt. Dabei hat er neben diversen Bildungsmodulen der IG Metall auch die Technologieberatungsstelle sowie die Hilfe gewerkschaftsnaher externer Berater in Anspruch genommen, die in diesen Prozessen eine zentrale Bedeutung erlangen können. So kann eine neue Qualität der Auseinandersetzung mit der Arbeitgeberseite über die Wege zur Erreichung bestimmter betriebswirtschaftlicher Ziele entstehen.

\subsection{EXTERNE BERATER}

Diese Ausweitung des strategischen Betriebsratshandelns setzt vielfach die Schulung der Betriebsräte voraus. Eine ganz wichtige Rolle dabei haben aber auch gewerkschaftsnahe Berater. Dies hat sich in den im vorigen Abschnitt geschilderten Fällen gezeigt. Berater können dabei je nach konkreter Fallkonstellation eine Vielzahl wichtiger Unterstützungsfunktionen übernehmen: Sie helfen dem Betriebsrat, Bilanzen und Kennziffern zu analysieren, erstellen im Rahmen von Tarifabweichungskonflikten Gutachten und führen Analysen durch, die anschließend als Grundlage für Verhandlungen mit der Geschäftsführung dienen, moderieren Gespräche zwischen Betriebsrat und Geschäftsführung, aber auch innerhalb des Betriebsrats, und planen und begleiten konkrete Verbesserungsprozesse. Eine wichtige Rolle spielt dabei die Gewerkschaft, die die Schulung und Beratung zum
Teil selbst ausführt, oftmals aber auch den Kontakt zu gewerkschaftsnahen externen Beratungsanbietern herstellt.

\subsection{ORGANISATION DES GREMIUMS}

Ein solches pro-aktives Handeln des Betriebsrates lässt sich - dies ist die zweite von uns beobachtete Tendenz - nicht (länger) als „One-Man-Show“ organisieren, bei der ein dominierender Betriebsratsvorsitzender eine klassische Stellvertreterpolitik betreibt. Vielmehr müssen Betriebsratsgremien zu arbeitenden Teams entwickelt werden. Beteiligungsorientierung fängt bereits innerhalb des Betriebsrats an. In vielen Fällen lässt sich zwar weiterhin eine starke Führungsfigur und eine aktive „Kerngruppe“ innerhalb des Betriebsrates identifizieren; trotzdem tendieren viele Betriebsratsgremien mittlerweile dazu, jüngere Betriebsräte verstärkt in die Verantwortung einzubinden, die Arbeitsteilung und Spezialisierung innerhalb des Gremiums zu erhöhen und zugleich den Kern der aktiven Betriebsräte zu erweitern. So werden beispielsweise in einem untersuchten Maschinenbaubetrieb speziell die jüngeren Betriebsratsmitglieder ausführlich zum Thema Gruppenarbeit geschult, damit diese bei der im Betrieb anstehenden Neuorganisation der Gruppenarbeit im Steuerungsgremium aktiv sein können. In einem anderen Betrieb wurden die jüngsten Neuwahlen genutzt, um insbesondere fachlich qualifizierte Angestellte für das Team zu gewinnen. In einem dritten Betrieb schließlich hat der Betriebsrat mittlerweile den verbindlichen Standard etabliert, dass freigestellte Betriebsräte eine Ausbildung zum Projektmanager bzw. Prozessbegleiter machen müssen, um fachlich wie methodisch in der Lage zu sein, Veränderungsprozesse aktiv mitzugestalten. Auch hier können externe Berater, aber auch die IG Metall mit ihren Schulungsangeboten, eine wichtige Rolle spielen.

\subsection{EXPERTENWISSEN DER BESCHÄFTIGTEN}

Die gezielte Beteiligung und Einbeziehung der Beschäftigten - und dies ist das dritte Element der Veränderung - spielt bei der Erarbeitung, Durchsetzung und Umsetzung von „Besser“-Konzepten eine zentrale Rolle. Bei der Verhandlung von Tarifabweichungen ist die Beteiligung der
Beschäftigten durch betriebliche Tarifkommissionen oder Mitgliederversammlungen mittlerweile verbreitete Praxis (vgl. Haipeter 2010); das Besondere von „Besser“-Strategien ist jedoch die gezielte Aktivierung und strategische Nutzung von Beschäftigtenwissen und Beschäftigtenkompetenz bei allen Fragen der Ausgestaltung der betrieblichen Arbeitsorganisation. Betriebsräte können aufgrund ihrer besonderen Vertrauensbeziehung zu den Beschäftigten einen anderen Zugang zum Erfahrungswissen der Beschäftigten finden als die Geschäftsführung. Zweitens können Betriebsräte dabei helfen, Akzeptanz und Legitimation für die Umsetzung geplanter Innovationen bei den Beschäftigten zu erzeugen (vgl. Schwarz-Kocher et al. 2010). Hinzu kommt, dass angesichts der häufigen personellen Wechsel in vielen Geschäftsleitungen der Betriebsrat zu einem Hort der Kontinuität im Betrieb wird, der gegenüber dem Management deutliche organisatorische und technologische Wissens- und Kompetenzvorsprünge hat.

Die Einbeziehung des Expertenwissens der Beschäftigten ist aber insbesondere auch für den Aufbau fachlicher Gegenmacht und die Entwicklung eigener (Alternativ-)Konzepte des Betriebsrates unverzichtbar. So werden u. a. Mitarbeiterbefragungen, Workshops und Gesprächskreise, „Klagemauern“ und ähnliche betriebsspezifische Kommunikationsinstrumente eingesetzt, um die Probleme, Vorstellungen und Wünsche der Beschäftigten abzufragen, Zielprioritäten festzulegen und die Belegschaft zugleich zur stärkeren Teilnahme zu motivieren. In fast allen von uns untersuchten Fällen sind die diesbezüglichen Initiativen des Betriebsrates von der Belegschaft ausgesprochen positiv aufgenommen worden. Im Rahmen von kontinuierlichen Verbesserungsprozessen und betrieblichem Vorschlagswesen können materielle Anreize dabei durchaus eine Rolle spielen; oftmals geht es für die beteiligten Beschäftigten aber auch um Fragen der Anerkennung und Wertschätzung der eigenen Kompetenzen. Ein interviewter Betriebsrat bringt es folgendermaßen auf den Punkt: „Das ist hier nicht immer nur Geld. Ich darf dem Chef das in die Augen sagen! Der Kollege, der hier die Präsentation gemacht hat, der war so stolz: Der Chef hat dabeigesessen, der Vorstand hat zugehört!“ Das strategische Problem, das sich hier jedoch abzeichnet, besteht darin, dass es immer stärker 
darauf ankommen wird, zusätzlich zur Kompetenz von Facharbeitern das Fachwissen von Ingenieuren zu mobilisieren - also einer Beschäftigtengruppe, in der die meisten Betriebsräte bislang schwach verankert sind. Trotzdem ist die Lage hierfür nicht aussichtslos. Das oben angeführte Fallbeispiel des Anlagenbauers zeigt, dass Betriebsräte über den Weg der fachlichen Anerkennung für ihre Alternativkonzepte Vertrauen in die Arbeit des Betriebsrats herstellen und neue Beschäftigtengruppen für sich gewinnen können.

\subsection{KONZEPT UND DRUCK}

Der Betriebsrat kann, wie bereits angedeutet, durch „Besser“-Initiativen seine Machtposition gegenüber der Unternehmensleitung stärken, weil er mithilfe eigener Vorschläge und neuer Unterstützung, sei es durch Beschäftigte, sei es durch externe Berater und die Gewerkschaft, nun „auf Augenhöhe" verhandelt. Dies gilt paradoxerweise auch für Vereinbarungen, die im Zusammenhang mit Tarifabweichungen abgeschlossen werden. In einigen Fällen ist es Betriebsräten in Tarifabweichungen gelungen, ihre Beteiligung an betrieblichen Veränderungsprozessen für die Zukunft formal festzuschreiben. Teilweise werden dabei auch Lernschleifen durchlaufen. So wurden bei einem Automobilzulieferer auf Drängen der Interessenvertretungen verbindliche Gespräche zwischen Management und Betriebsrat über Verbesserungen der Organisation und Maßnahmen zur Steigerung der Produktivität in der Tarifabweichung festgeschrieben. Weil die Geschäftsleitung gegen diese Vorgabe dann anhaltenden Widerstand leistete, wurde in der darauf folgenden Tarifabweichung die Verbindlichkeit dieser Gespräche erhöht, indem ein gemeinsamer Lenkungskreis geschaffen und zudem das Unternehmen verpflichtet wurde, einen externen gewerkschaftsnahen Berater einzustellen. Als auch dies nicht zu zufriedenstellenden Ergebnissen führte, haben die Interessenvertretungen schließlich die Sitzungsfristen des Lenkungskreises verkürzt und eine Berichtspflicht nach außen gegenüber der IG Metall festschreiben können, die mehrmals jährlich über Probleme und Fortschritte zu unterrichten ist. Auf diesem Wege können dem Betriebsrat per Betriebsvereinbarung oder abweichendem Tarifvertrag Einflussmöglichkeiten auf betriebliche Innovationsprozesse rechtlich gesichert werden, die über die Regelungen des Betriebsverfassungsgesetzes deutlich hinausgehen.

Wie unsere Fallstudien zeigen, sind „Konzept und Druck“ (Korflür et al. 2010) zwei Seiten einer Medaille in betrieblichen „Besser“-Prozessen. Es bleibt unverzichtbar, dass der Betriebsrat in jeder Phase des Prozesses die Belegschaft mobilisieren kann. So haben in einem Fall erst Warnund Bummelstreiks der Belegschaft, die zwischenzeitlich zu erheblichen finanziellen Belastungen für das Unternehmen führten, den nötigen Druck erzeugt, um die Geschäftsführung überhaupt zur Aufnahme von Verhandlungen zu bewegen. In einem anderen Fall haben sich massenhafte „Besuche beim Betriebsrat", die den laufenden Betrieb zum Stillstand brachten, als wichtige Drohkulisse während der Verhandlungen mit der Geschäftsführung erwiesen. Wie sich in einem weiteren Fall zeigte, kann es auch nach Abschluss der Verhandlungen notwendig sein, durch die Drohung mit der Kündigung der ausgehandelten Vereinbarung den Druck aufrecht zu erhalten, wenn die Unternehmensleitung bestimmte Punkte der Vereinbarung nicht oder nur zögerlich umsetzt.

\section{Schlussfolgerungen}

Die gestaltungs- und beteiligungsorientierten Ansätze, die mit Kampagnen wie „Besser statt billiger" gefördert werden sollen, sind nach unseren Beobachtungen mit Brüchen für das Selbstverständnis und die Organisation der Interessenvertretungen verbunden. Dabei geht es nicht um einen Ersatz, sondern um eine Ergänzung und Erweiterung der klassischen Betriebsratsarbeit. Betriebsräte, die sich in der konventionellen Rolle des „Schützers und Bewahrers" sehen, stoßen nach unserem Eindruck vermehrt an Grenzen. Ähnliches gilt für Betriebsräte, die sich eher als konfliktorientierte Verteidiger von Beschäftigteninteressen verstehen, ohne sich dabei „den Kopf des Arbeitgebers zerbrechen zu müssen“. Wenn die Unternehmen ihre „Bargaining Chips“ ausspielen, die sie als Drohpotenziale trotz der Finanzmarktkrise mit Verweis auf Auslagerungsmöglichkeiten und die internationale Standortkonkurrenz nach wie vor haben, müssen die Betriebsräte Gegenkonzepte entwickeln und überzeugend vertreten können, um in diesen De- fensivkonstellationen noch - oder wieder - auf Augenhöhe mit dem Management verhandeln zu können.

Unsere ersten Eindrücke aus betrieblichen Fallstudien zeigen, dass Interessenvertretungen auch in dieser Defensivsituation durchaus über erhebliche Handlungsspielräume und Gestaltungspotenziale verfügen - allerdings nur um den Preis der Veränderung ihres Selbstverständnisses. $\mathrm{Zu}$ bedenken ist dabei allerdings, dass mit „Besser“-Konzepten nicht alle Probleme gelöst sind, sondern auch neue Probleme entstehen können. Zum einen werden Defensivkonstellationen nicht aufgelöst. Auch im Erfolgsfall werden nur temporäre arbeitspolitische Akzente gesetzt. Die innovative Arbeitspolitik bleibt eine Daueraufgabe, weil die Drohkulissen des Managements bestehen bleiben und jederzeit der arbeitspolitische Rückschritt droht. Deshalb ist sie auch immer an die Verteidigung der bestehenden Arbeitsstandards und damit an eine Schutzpolitik der Interessenvertretungen gebunden. Zum anderen erweist sich die praktische Verknüpfung von betrieblichen Innovationen, die von Betriebsräten als Alternative zu Tarifabweichungen oder Auslagerungen angestoßen werden, und "guter Arbeit", also erfahrbaren Aufwertungen der Beschäftigten im Arbeitsprozess, als wenig erkundetes Neuland. Es zu erschließen, wird jedoch notwendig sein, wenn aus einzelnen betrieblichen Verbesserungsinitiativen eine Strategie entstehen soll, die auf eine Revitalisierung des deutschen Produktionsmodells durch tatsächliche Nutzung der ihm zugeschriebenen Stärken abzielt.

Die gegenwärtige Krise bietet paradoxerweise neue Anknüpfungspunkte für arbeitspolitische Innovationen der Interessenvertretungen, weil in vielen Betrieben mit dem „Horten von Arbeitskräften“ erstmalig seit langer Zeit die Bedeutung qualifizierter Beschäftigter auch praktisch anerkannt wurde. Diese Chance, sich gegen die zu erwartende Hinwendung zu verschärften Umstrukturierungsmaßnahmen in Stellung zu bringen, ist die Herausforderung, der sich Gewerkschaften und Betriebsräte jetzt mehr und mehr gegenübersehen. Die Erfahrungen, die im Zusammenhang mit „Besser statt billiger" und vergleichbaren Projekten gesammelt wurden, sind ein Schatz, den zu heben sich für die Bewältigung dieser Herausforderung lohnen wird. 
Boyer, R. (2010): The Collapse of Finance but Labour Remains Weak, in: Socio-Economic Review 2, S. 348-353

Crouch, C. (2010): The Financial Crisis a new Chance for Labour Movements? Not Yet, in: Socio-Economic Review 2, S. 353-356 Dörre, K./Holst, H.(2009): Nach dem Shareholder Value? Kapitalmarktorientierte Unternehmenssteuerung in der Krise, in: WSI-Mitteilungen 12 , S. 667-674

Ehlscheid C./Pickshaus K./Urban H.-J. (2010): Die große Krise und die Chancen der Gewerkschaften, in: Sozialismus 6, S. 43-49

Haipeter, T. (2010): Betriebsräte als neue Tarifakteure. Zum Wandel der Mitbestimmung bei Tarifabweichungen, Berlin

Hall, P./Soskice, D. (2001): An Introduction to Varieties of Capitalism, in: Hall, P./Soskice, D. (Hrsg.): Varieties of Capitalism: The Institutional Foundations of Comparative Advantage, Oxford/ New York, S. 1-71

Herzog-Stein, A. (2010): Das Jahr der Krise. Ein Vergleich verschiedener Abschwungphasen seit 1970, in: Groß, H./Seifert, H. (Hrsg.): Zeitkonflikte. Renaissance der Arbeitszeitpolitik, Berlin, S. 17-36

Kirner, E./Weißfloch, U./Jäger, A. (2010): Beteiligungsorientierte Organisation und Innovation, in: WSI-Mitteilungen 2, S. 87-94 Korflür, I./Nettelstroth, W./Schilling, G./Schlette, M./Vanselow, A. (2010): „Besser statt billiger“ im Betrieb, in: WSI-Mitteilungen 2, S. $109-112$
Kuhlmann, M. (2009): Perspektiven der Arbeitspolitik nach der Krise Entwicklungslinien und Handlungsbedingungen, in: WSI-Mitteilungen 12, S. $675-682$

Lehndorff, Steffen, (Hrsg) (2009): Abriss, Umbau, Renovierung? Studien zum Wandel des deutschen Kapitalismusmodells, Hamburg Schroth, J./Wetzel, D. (2009): Gegenwärtige arbeitspolitische Herausforderungen sowie Schwerpunkte und Planungen der IG Metall - Besser statt billiger als betriebspolitisches Konzept, Referat im Arbeitskreis Arbeitspolitik, 25. September, IG Metall Vorstand, Frankfurt (unveröffentlichtes Manuskript)

Schumann, M. (2001): Kritische Industriesoziologie - Neue Aufgaben, in: SOFI-Mitteilungen 29, S. 93-97

Schumann, M. (2008): Kampf um Rationalisierung - Suche nach neuer Übersichtlichkeit, in: WSI-Mitteilungen 7, S. 379-386

Schwarz-Kocher, M./Dispan, J./Richter, U./Seibold, B. (2010): Betriebsratshandeln im Modus arbeitsorientierter Innovationsprozesse, in: WSI-Mitteilungen 2, S. 95-102

Seifert, H. (2010): Beschäftigungssichernde Arbeitszeitverkürzungen Intermezzo oder arbeitszeitpolitischer Aufbruch, in: Groß, H./Seifert, H. (Hrsg.): Zeitkonflikte. Renaissance der Arbeitszeitpolitik, Berlin, S. 37-52 Urban, H.-J. (2008): Vorwort, in: Brinkmann, U./Choi, H./Detje, R./ Dörre, K./Holst, H./Karakayali, S./Schmalstieg, C.: Strategic Unionism. Aus der Krise zur Erneuerung?, Wiesbaden, S. 7-13 